\title{
ZEB1 transcriptionally regulated carbonic anhydrase 9 mediates the chemoresistance of tongue cancer via maintaining intracellular $\mathrm{pH}$
}

Guopei Zheng ${ }^{1 \dagger}$, Cong Peng ${ }^{1 \dagger}$, Xiaoting Jia ${ }^{1}$, Yixue Gu${ }^{1}$, Zhijie Zhang ${ }^{1}$, Yingen Deng ${ }^{1}$, Chengkun Wang ${ }^{1}$, Nan $\mathrm{Li}^{1}$, Jiang Yin', Xiaorong Liu', Minying Lu' ${ }^{1}$, Hailin Tang ${ }^{2}$ and Zhimin $\mathrm{He}^{1^{*}}$

\begin{abstract}
Background: Chemoresistance is a major obstacle in successfully treating cancers, and the mechanisms responsible for drug resistance are still far from understood. Carbonic anhydrase 9 (CA9) has been shown to be upregulated in the drug-resistant tongue cancer cell line Tca8113/PYM and to be associated with drug resistance. However, the mechanisms regulating CA9 expression and its role in drug resistance remain unclear.

Methods: Bioinformatic and experimental analysis involving ChIP and luciferase reporter assays were used to validate Zinc finger E-box-binding homeobox 1 (ZEB1) as a transcriptional regulator of CA9. Gene expression and protein levels were evaluated by quantitative RT-PCR and western blotting, respectively. Sensitivity to chemotherapy was examined using the MTS assay and Hoechst staining and analysis caspase-3 activity to evaluate changes in apoptosis. Intracellular pH (pHi) was measured using fluorescent pH-indicator BCECF-AM. Protein expression in patient tissue samples was examined by immunohistochemistry and survival of tongue cancer patients from which these samples were derived was also analyzed.
\end{abstract}

Results: ZEB1 bound to the promoter of CA9 to positively regulate CA9 expression in tongue cancer cells. Knockdown of CA9 using short interfering RNA (siRNA) abolished the chemoresistance resulting from ZEB1 overexpression in Tca8113 and SCC-25 cells, and CA9 overexpression attenuated chemosensitivity induced by ZEB1 knockdown in Tca8113/PYM cells. CA9 knockdown also prevented maintenance of pHi mediated by overexpression of ZEB1 in Tca8113 and SCC-25 cells following chemotherapy, associated with increased apoptosis and caspase-3 activation. Conversely, ectopic expression of CA9 suppressed decrease in pHi mediated by ZEB1 knockdown in Tca8113/PYM cells following chemotherapy, accompanied by decreased apoptosis and caspase-3 activation. Importantly, a positive correlation was observed between ZEB1 and CA9 protein expression in tongue cancer tissues, and expression of these proteins associated with a poor prognosis for patients.

Conclusion: Our finding that tumor cells regulate $\mathrm{pHi}$ in response to chemotherapy provides new insights into mechanisms of drug resistance during cancer treatment. Identification of the ZEB1-CA9 signaling axis as a biomarker of poor prognosis in tongue cancer will be valuable in future development of therapeutic strategies aimed at improving treatment efficacy, especially in terms of drug resistance associated with this disease.

Keywords: Carbonic anhydrase 9, ZEB1, Intracellular pH, Tongue cancer, Chemoresistance

\footnotetext{
*Correspondence: hezhimin2005@yahoo.com

'Equal contributors

'Cancer Hospital and Cancer Research Institute of Guangzhou Medical

University, Hengzhigang Road 78\#, Guangzhou 510095, Guangdong, China

Full list of author information is available at the end of the article
}

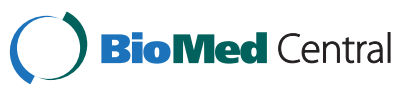

(C) 2015 Zheng et al.; licensee BioMed Central. This is an Open Access article distributed under the terms of the Creative Commons Attribution License (http://creativecommons.org/licenses/by/4.0), which permits unrestricted use, distribution, and reproduction in any medium, provided the original work is properly credited. The Creative Commons Public Domain Dedication waiver (http://creativecommons.org/publicdomain/zero/1.0/) applies to the data made available in this article, unless otherwise stated. 


\section{Background}

Squamous cell carcinoma (SCC) of the oral cavity represents the tenth most frequent solid cancer worldwide [1]. Tongue cancer is the most common type of oral cancer and frequently leads to the malfunction of mastication, speech, and deglutition [2]. As one of the standard therapeutic approaches, chemotherapy mostly based on pingyangmycin (PYM) and/or cisplatin (cDDP), plays an important role in tongue cancer treatment and brings many benefits including reducing tumor size, inhibiting distant metastasis and prolonging patient survival [3]. However, resistance to anticancer agents is a major obstacle for successful chemotherapy in tongue cancer, as it can result in more aggressive tumor behavior and worse clinical outcome $[4,5]$. The molecular basis of sensitivity and resistance to chemotherapy is complex, involving multiple biological processes such as drug transport, drug metabolism, apoptosis and DNA repair [6]. Although the mechanisms responsible for drug resistance in cancer have been the subject of intense studies for decades, the clinical causes of drug resistance remain poorly understood. There is, therefore, an urgent need to clarify these mechanisms and to explore alternative therapeutic strategies to overcome drug resistance in tongue cancer treatment.

We have previously shown that carbonic anhydrase IX (CA9) expression is increased in the pingyangmycin (PYM)-induced multidrug-resistant human tongue squamous cell carcinoma cell line Tca8113/PYM when compared to the parental cell line Tca8113, without significant expression difference in multidrug resistance genes such as MDR, BCRP and MRP. Functional experiments demonstrated that both carbonic anhydrase inhibitor application and knockdown of CA9 expression enhanced PYM chemosensitivity [7]. In agreement with our previous study, Michael et al. have suggested that CA9 expression in squamous cell head and neck tumors is associated with resistance to chemoradiotherapy [8]. CA9 overexpression has been identified in a number of solid tumors, including renal carcinomas, cervical squamous carcinomas, esophageal carcinomas, bladder carcinomas and non-small cell lung carcinomas. CA9 as a membrane-associated protein is a zinc metalloenzyme catalyzing the reversible conversion of $\mathrm{CO}_{2}$ to bicarbonate and a proton, and this has the effect of reducing local extracellular $\mathrm{pH}$ [9]. Under equivalent culture conditions, the extracellular $\mathrm{pH}(\mathrm{pHe})$ of the culture media of Tca8113/PYM cells is much lower than that found for the culture media of Tca8113 cells, and this can be attributed to the increased expression of CA9 in the Tca8113/PYM cell line [7]. Generally, solid cancers maintain a high intracellular $\mathrm{pH}(\mathrm{pHi})$ but a low extracellular $\mathrm{pH}(\mathrm{pHe})$ that results from hypoxia or acidosis. Adaptation of cancer cells to hypoxia and acidosis is a critical driving force in tumor progression and metastasis $[10,11]$. Cancer cells have developed key strategies to regulate their $\mathrm{pHi}$, because a $\mathrm{pHi}$ variation of as little as 0.1 can disrupt multiple biological functions including ATP production, protein synthesis, cell proliferation, migration and apoptosis [12-14]. Whether cellular adaptation associated with drug resistance is due to CA9 overexpression requires further investigation. CA9 expression is strongly induced by hypoxia-inducible factor 1 (HIF-1) and is believed to be involved in cancer cell proliferation, transformation and survival, making it a potential target for cancer therapy $[15,16]$. However, there is no significant difference in the expression of HIF-1 between Tca8113 and Tca8113/PYM cells, implying some other factors are responsible for CA9 overexpression in Tca8113/PYM cells. In the present study, we investigated the expression patterns of, and correlation in expression between, CA9 and ZEB1 in tongue cancer cell lines and tissues. Bioinformatics analysis combined with experimental validation demonstrated that ZEB1 transcriptionally regulates CA9 expression and that ZEB1 may contribute to chemotherapy resistance via CA9-dependent modulation of $\mathrm{pHi}$.

\section{Results}

\section{ZEB1 transcriptionally regulates CA9 expression in tongue} cancer cells

We have previously shown that both CA9 mRNA and protein expression is upregulated in the PYM-induced multidrug-resistant tongue cancer cell line Tca8113/PYM. To determine whether transcriptional regulation contributes to CA9 upregulation in Tca8113/PYM cells, we analyzed the response elements of a cohort of transcription factors located within a three kilobase region upstream of the first exon of the CA9 gene. Using the JASPAR database (http://jaspar.binf.ku.dk) we identified five putative ZEB1 binding sites within this region, conforming to the optimal recognition sequence of ZEB1 (CACCTG) (Figure 1A). To confirm the direct association of ZEB1 with the CA9 promoter, we performed a ChIP assay in Tca8113/PYM cells for all putative ZEB1 binding sites within the three kilobase region. ChIP results revealed that ZEB1 bound most significantly to sites $\mathrm{B}, \mathrm{C}$ and $\mathrm{E}$ within the potential CA9 promoter (Figure 1B). As expected, ectopic expression of ZEB1 using the pLEX-ZEB1 construct enhanced both CA9 mRNA and protein expression in Tca8113 and SCC-25 cell lines (Figure 1C and D). Conversely, CA9 mRNA and protein expression decreased following knockdown of ZEB1 in Tca8113/PYM cells using ZEB1-specific siRNAs (Figure $1 \mathrm{C}$ and $\mathrm{D}$ ). To investigate further the effects of ZEB1 on CA9 expression, the putative three kilobase $C A 9$ promoter was cloned into a luciferase reporter vector and expression assays subsequently performed. As expected, CA9 promoterdriven luciferase activity was much higher in Tca8113/PYM cells than in Tca8113 and SCC-25 cells (Figure 1E). In 


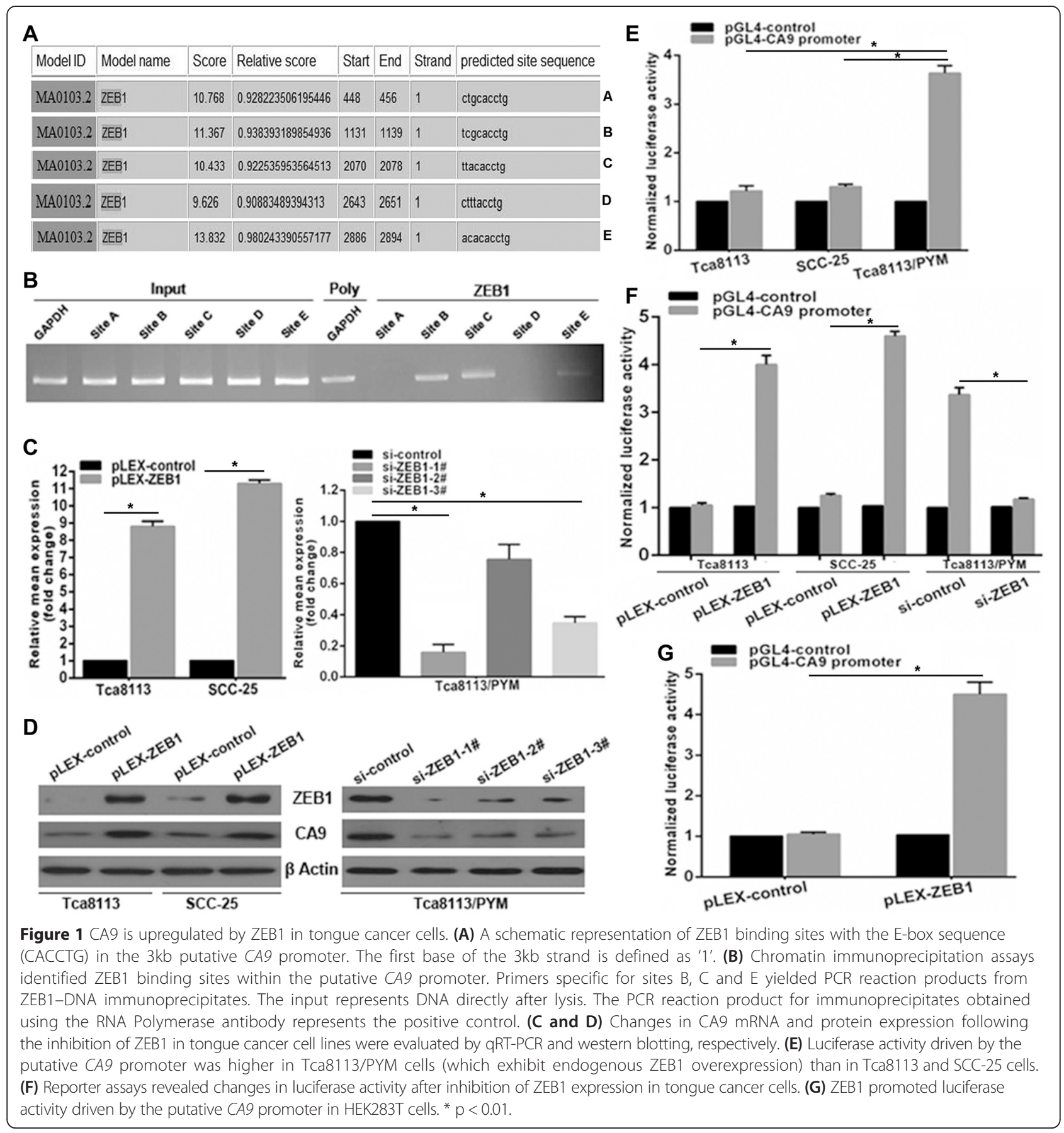

addition, ZEB1 was found to significantly enhanced luciferase activity driven by the CA9 promoter in HEK293T cells (Figure 1G). These results demonstrate that ZEB1 can directly bind to the $C A 9$ promoter to transcriptionally regulate $\mathrm{CA} 9$ expression.

\section{CA9 contributes to ZEB1-mediated drug resistance in tongue cancer cells}

We have previously shown that CA9 is involved in drug resistance in tongue cancer cells, and have shown here that ZEB1 positively regulates CA9 expression. Next, we wished to determine whether ZEB1 modulates chemosensitivity in tongue cancer and whether it exerts its effect via regulating CA9 expression. We found that ectopic ZEB1 expression enhanced the resistance of Tca8113 and SCC25 cells to PYM or cDDP, with the marked increase of IC50 values (Figure 2A and B). Conversely, ZEB1 knockdown increased the sensitivity of Tca8113/PYM cells to PYM and cDDP, with the significant decrease of IC50 values (Figure 2C). Moreover, CA9 knockdown markedly 


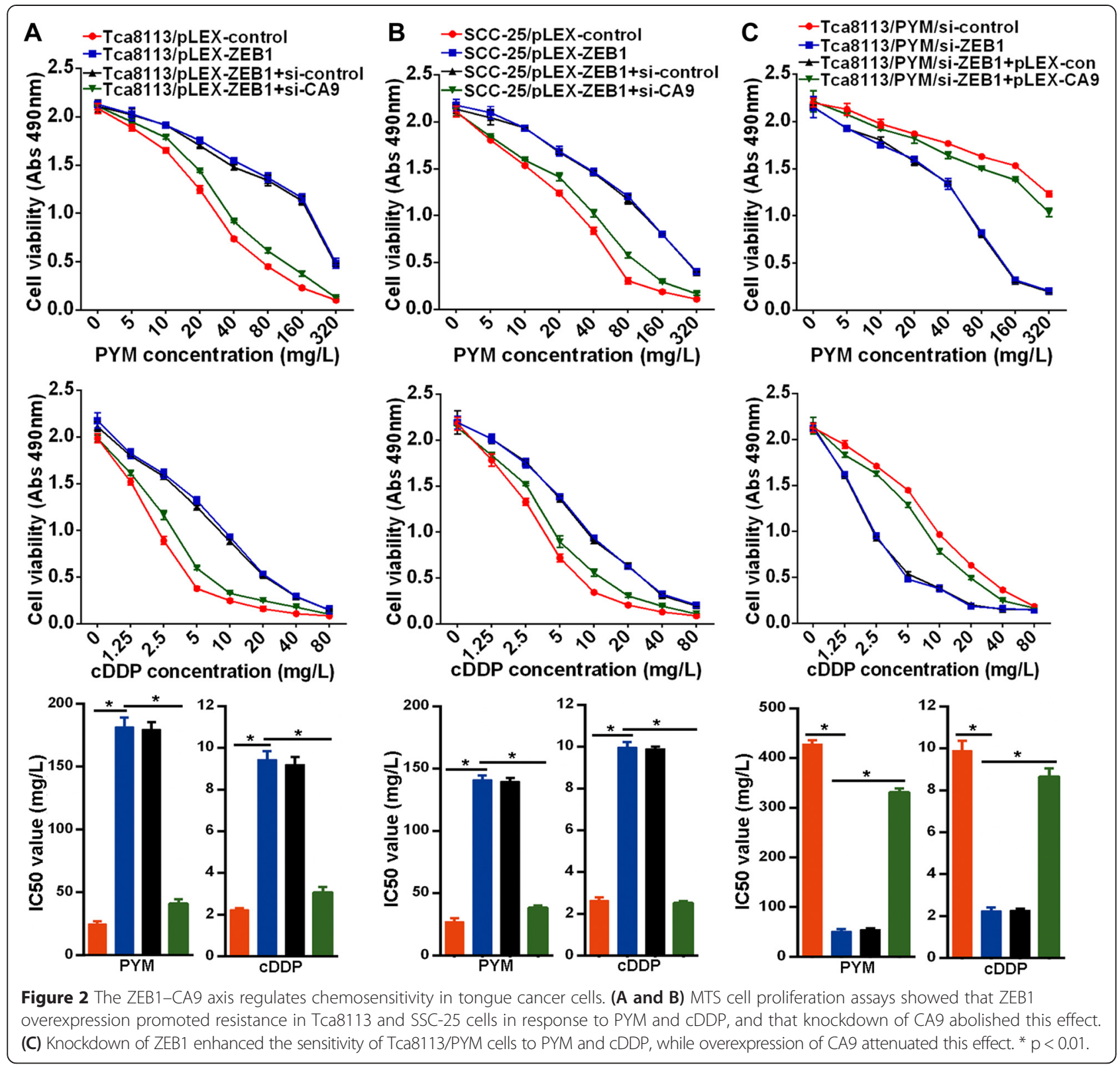

impaired ZEB1-mediated drug resistance to PYM or cDDP in Tca8113 and SCC-25 cells (Figure 2A and B), while overexpression of CA9 in Tca8113/PYM cells impaired the effect of ZEB1 knockdown in response to chemotherapy (Figure 2C), suggesting CA9 is responsible for ZEB1mediated drug resistance in tongue cancer cells.

The ZEB1-CA9 axis regulates chemotherapy-induced changes in intracellular $\mathrm{pH}$

Tumor cells usually have a higher pHi (which is neutralto-alkaline) in comparison to normal cells [17]. Alterations in $\mathrm{pHi}$ homeostasis have been implicated in anticancer drug treatment and drug-resistant cancer cells may develop multiple mechanisms to regulate $\mathrm{pHi}$ more effectively in response to extracellular or intracellular stress. Here, we found that PYM $(80 \mathrm{mg} / \mathrm{L})$ or cDDP $(5 \mathrm{mg} / \mathrm{L})$ treatment resulted in a significant decrease in the $\mathrm{pHi}$ (Figure $3 \mathrm{~A}$ and $\mathrm{C}$ ) and an accompanying increase in apoptosis (Figure 3B and D) in Tca8113 and SCC-25 cells when compared to Tca8113/PYM cells (Figure 3E and F). Ectopic ZEB1 expression markedly impaired the PYM or cDDP-induced decrease in $\mathrm{pHi}$ and the associated increase in apoptosis in Tca8113 and SCC-25 cell lines (Figure 3A-D). However, knockdown of CA9 in these cells attenuated the effect of ectopic ZEB1 expression on pHi and apoptosis following PYM or cDDP treatment (Figure 3A-D). Moreover, in Tca8113/PYM cells, knockdown of ZEB1 enhanced the PYM or cDDP-dependent 


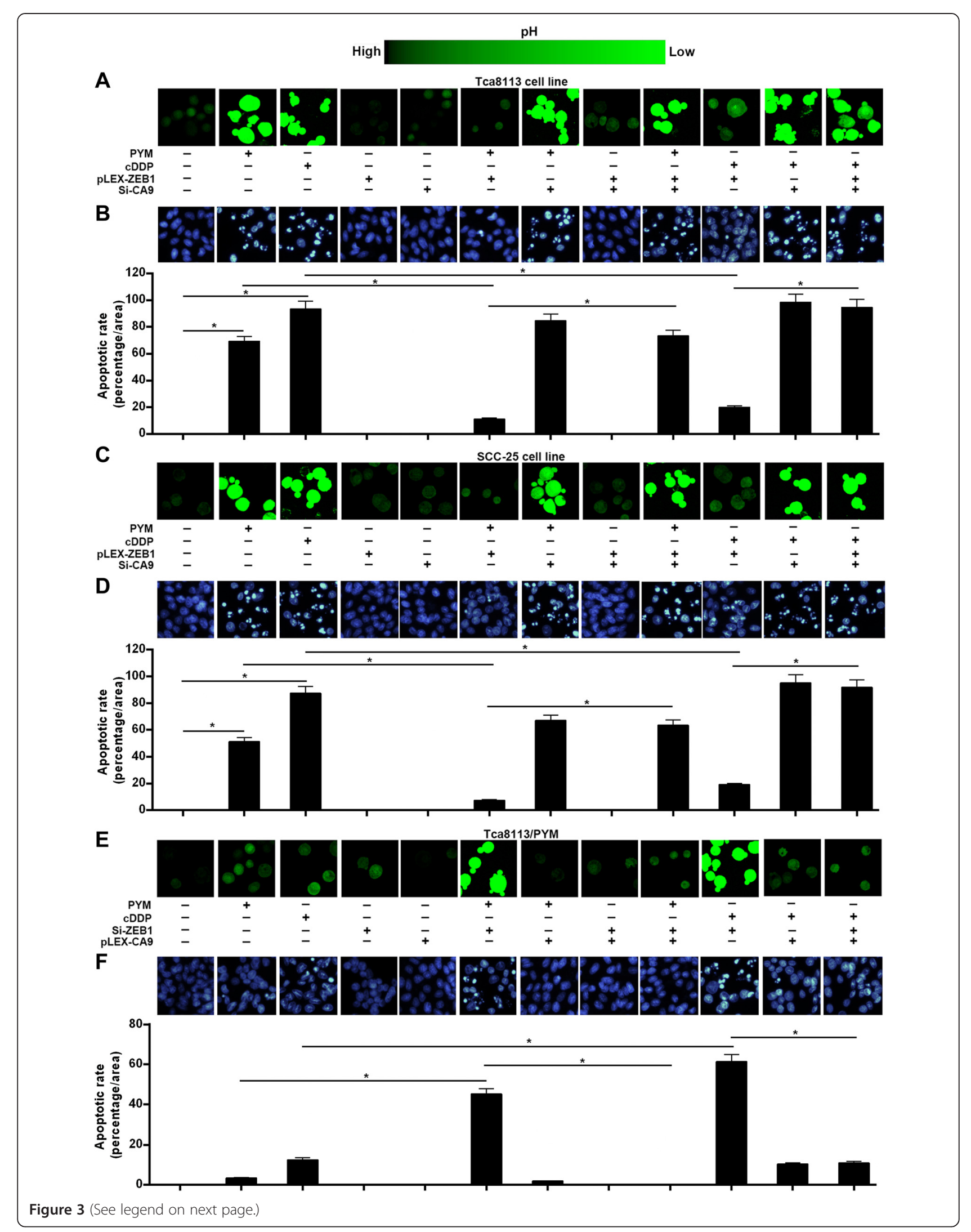


(See figure on previous page.)

Figure 3 The ZEB1-CA9 axis prevents pHi decrease induced by chemotherapy in tongue cancer cells. (A, C and E) The change in pHi found for each given cell line in response to PYM or CDDP treatment, as determined using the BCECF-AM pH fluorescence probe in conjunction with confocal microscopy. (B, D and F) Changes in apoptosis in response to PYM or CDDP treatment for each given cell line, as determined by Hoechst staining evaluated by fluorescence microscopy. ${ }^{*} p<0.01$.

decrease in $\mathrm{pHi}$ and the associated increase in apoptosis, while overexpression of CA9 essentially abolished these effects (Figure 3E and F). These findings suggest that the ZEB1-CA9 signaling axis prevents pHi decrease and apoptosis induced by chemotherapy.

\section{The ZEB1-CA9 axis modulates chemotherapy-induced caspase-3 activation}

A decrease in pHi correlates with an increase in apoptosis and caspase- 3 is a key component of the apoptotic pathway [18]. We therefore performed a caspase- 3 activity assay and western blotting to detect the cleaved, active protein and therefore determine the functional state of caspase- 3 in cultured cells. We found that PYM $(80 \mathrm{mg} / \mathrm{L})$ or $\mathrm{cDDP}$ $(5 \mathrm{mg} / \mathrm{L})$ treatment significantly promoted caspase-3 activation in Tca8113 and SCC-25 cells (Figure 4A and B) and this was accompanied by a decrease in pHi (Figure 3A and $\mathrm{B})$. With respect to the change in $\mathrm{pHi}$, we found that ectopic ZEB1 expression blocked PYM or cDDP-induced caspase-3 activation in Tca8113 and SCC-25 cells (Figure $4 \mathrm{~A}$ and $\mathrm{B}$ ), while the simultaneous knockdown of CA9 abolished this ZEB1-related effect (Figure 4A and B). Furthermore, in Tca8113/PYM cells (in which endogenous ZEB1 is upregulated) we found that knockdown of ZEB1 promoted caspase-3 activation in response to PYM or cDDP (Figure 4C). However, ectopic CA9 expression attenuated the effect of ZEB1 knockdown on caspase-3 activation (Figure 4C). These results suggest that CA9 is involved in the ZEB1-dependent inhibition of caspase-3 activation.

\section{ZEB1 and CA9 are negative prognostic markers in tongue cancer}

Given the above observations in cultured cells, namely that ZEB1 transcriptionally regulates CA9 expression and that the ZEB1-CA9 axis mediates chemotherapy resistance, we next wished to assess whether there is a correlation between ZEB1 expression and CA9 expression in tongue cancer. We therefore performed immunohistochemical staining on tissue arrays containing tongue cancer tissue from 84 patients receiving chemotherapy based on PYM and/or cDDP. Notably, CA9 protein was strongly expressed in 39 of 41 tongue cancer tissues that exhibited high ZEB1 protein expression (Figure 5A). In contrast, CA9 protein was strongly expressed in only 8 of 43 tongue cancer tissues that exhibited low ZEB1 protein expression (Figure 5A). Importantly, tongue cancer patients with high ZEB1 expression have a poor prognosis for overall survival compared to patients with low ZEB1 expression (Figure 5B).
In addition, high CA9 expression was also predictive of worse overall survival for tongue cancer patients (Figure 5B). Moreover, tongue cancer patients with both high ZEB1 and CA9 expression have worse overall survival compared to patients with both low ZEB1 and CA9 expression (Figure 5B). All the results denoted a potential correlation between ZEB1 and CA9 expression and their indication for the poor prognosis in human tongue cancer patients.

\section{Discussion}

Therapeutic resistance, which often appears and which is caused by various mechanisms, is the central problem in cancer therapy [19]. This underlines the critical importance of elucidating the precise molecular mechanisms involved in the anticancer drug resistance of cancer cells for improving current treatments in the clinic. We have previously shown that overexpression of CA9 is associated with drug resistance in tongue cancer cells [7], although at that time the mechanism of CA9 upregulation was unclear. In the present study, bioinformatics analysis combined with experimental validation has revealed that ZEB1 binds to the promoter of the CA9 gene and positively regulates its activity. ZEB1 expression was also found to promote CA9 expression in tongue cancer cells. Given the role of CA9 in drug resistance and that ZEB1 regulates the expression of CA9, we further investigated the role of ZEB1 in the drug resistance of tongue cancer cells. We found that ectopic expression of ZEB1 in tongue cancer cells significantly enhanced their resistance to chemotherapy while knockdown of ZEB1, conversely, increased sensitivity to chemotherapy. Moreover, knockdown of CA9 attenuated the effect of ectopic ZEB1 expression on the chemosensitivity of Tca8113 and SCC-25 cell lines and ectopic CA9 expression abolished the effect of ZEB1 knockdown on chemoresistant Tca8113/PYM cells.

ZEB1 has been reported to be highly expressed in epithelial cancers and its expression correlates with poor prognosis [20]. ZEB1 is a known driver of epithelialto-mesenchymal transition (EMT), a phenotype associated with cancer cells that are typically prone to metastasis, drug resistance and poor clinical outcome, and which represent potential therapeutic targets for cancer progression. ZEB1 represses the expression of epithelial genes and certain micro RNAs (miRNAs), including the miR-183, miR-203 and miR-200 family members, which function not only as strong inducers of EMT but also as inhibitors 


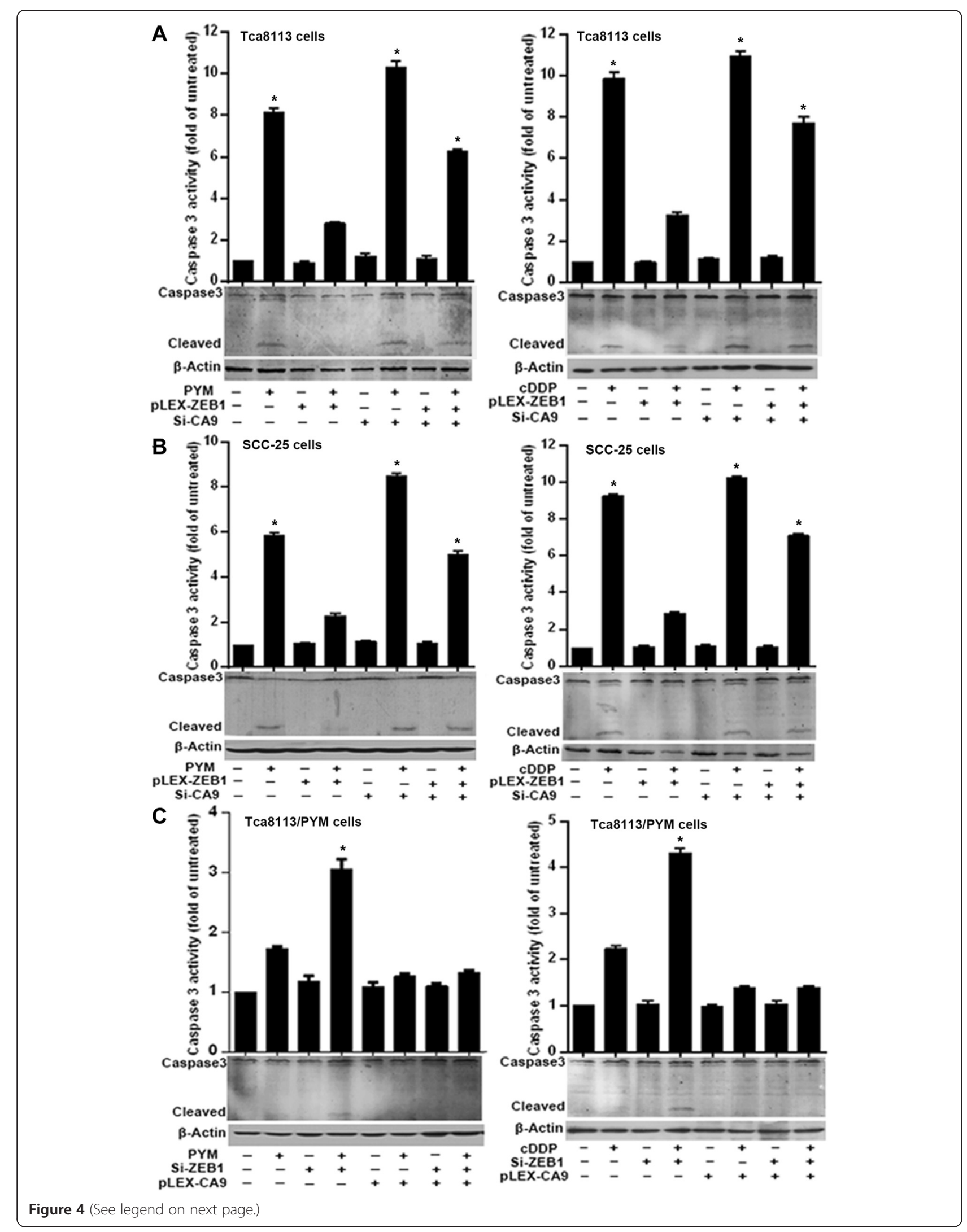


(See figure on previous page.)

Figure 4 ZEB1-CA9 prevents chemotherapy-induced caspase-3 activation. (A and B) PYM and cDDP induced caspase-3 activation in Tca8113 and SCC-25 cell lines, respectively, as measured by reporter assays and western blotting. Overexpression of ZEB1 prevented caspase-3 activation in response to chemotherapy, while knockdown of CA9 impaired the effects of ZEB1 overexpression. (C) Chemotherapy had no significant effect on caspase-3 activation in Tca8113/PYM cells. Knockdown of ZEB1 enhanced caspase-3 activation induced by chemotherapy, and overexpression of CA9 attenuated this effect. vs. no treatment, ${ }^{*} p<0.01$.

of stem cell properties [21,22]. ZEB1 knockdown reverses the EMT phenotype, inhibits migratory ability and enhances the chemosensitivity of docetaxel-resistant human lung adenocarcinoma cells [23]. The ZEB1 pathway links glioblastoma initiation and invasion. ZEB1 expression in glioblastoma patients is predictive of shorter survival and poor temozolomide response [24]. Moreover, ZEB1 has also been reported to be closely associated with radioresistance $[25,26]$. We now show here that ZEB1 predominantly correlates with drug resistance, mainly via modulating CA9 expression in tongue cancer cells.
CA9 has been reported to be overexpressed in many types of solid tumors and contributes to low pHe [9], as observed in our experimental model [7]. The cancer cells of solid tumors usually developed the ability to maintain a high $\mathrm{pHi}$ and a low pHe to adapt and sustain normal biological process, as intracellular acidification is closely associated with apoptosis. A change of $\mathrm{pHi} / \mathrm{pHe}$ ratio of 0.1-0.2 pH units can have disastrous consequences for critical biological processes including ATP synthesis, proliferation, metastasis and survival [27-29]. Nitric oxideinduced acidification drives nitric oxide-induced apoptosis

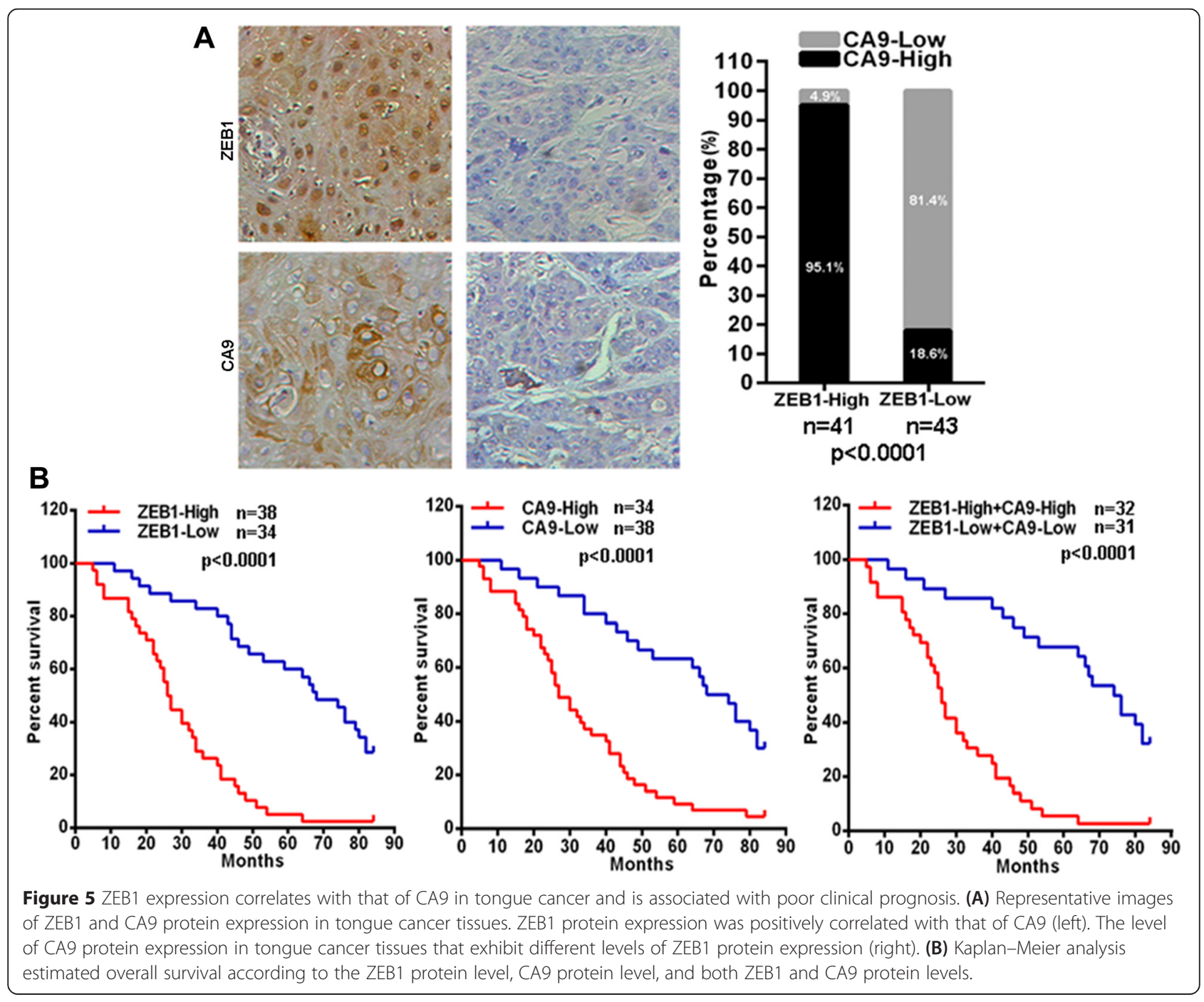


in neurons [30]. Studies from Rich et al. have shown that leukemic cell lines and peripheral blood from primary patient leukemic samples exhibited a constitutively higher $\mathrm{pHi}$ than normal hematopoietic tissue, and that a pHi decrease enhanced apoptosis in leukemic cells [18]. Caspase activation plays a critical role during apoptosis. The efficiency of caspase activation by cytochrome $\mathrm{C}$ is $\mathrm{pH}$-sensitive, and lower $\mathrm{pH}$ drives this activation [31]. With respect to CA9, Chiche et al. have shown that forced expression of CA9 contributes to extracellular acidification and to the maintenance of a more alkaline pHi [32]. The primary enzymatic function of CA9 is to catalyze the reversible hydration of carbon dioxide to bicarbonate and protons to contribute to acidification of the tumor microenvironment, acting as a "catalytic converter" for the excretion of acid from cells. Bicarbonate is shuttled into the cytoplasm to buffer $\mathrm{pHi}$, while the proton remains in the extracellular space. The biological effects of CA9 help to produce and maintain an alkaline $\mathrm{pHi}$ favorable for tumor growth, and participate in the generation of an increasingly acidic extracellular space, facilitating tumor progression. Thus, Inhibition of CA9 interferes with removal of acid and results in a decrease in pHi, negatively influencing cell survival $[33,34]$. In our study here we performed a series of experiments to determine whether ZEB1-CA9 mediates drug resistance via maintaining the $\mathrm{pHi}$. We found that the pHi in chemo-sensitive Tca8113 and SCC25 cells rapidly decreased upon treatment with PYM or cDDP, whereas this was not observed for chemoresistant Tca8113/PYM cells. However, overexpression of ZEB1 prevented both the pHi decrease and caspase- 3 activation induced by chemotherapy, and knockdown of CA9 abolished the effect of ZEB1 overexpression on Tca8113 and SCC-25 cells. In multidrug-resistant Tca8113/PYM cells, knockdown of ZEB1 promoted a decrease in $\mathrm{pHi}$ after exposure to PYM or cDDP, but this decrease was blocked following CA9 overexpression. Importantly, we found that ZEB1 protein expression strongly correlated with CA9 protein expression, and that the ZEB1-CA9 axis was a negative prognostic factor for overall survival in tongue cancer patients. Therefore, ZEB1 transcriptionally regulates CA9 expression to mediate chemoresistance in tongue cancer via modulating $\mathrm{pHi}$ in response to chemotherapy. Further study will now be required to elucidate the molecular mechanisms coupling ZEB1-CA9 to changes in pHi and the response to chemotherapy, and to determine whether observations made in vitro are reflected in the clinic. In addition, new strategies should be developed to enable the monitoring of these biochemical changes in response to chemotherapeutic agents both in vitro and in vivo.

\section{Conclusion}

In our present study, we elucidated the mechanisms responsible for the regulation of CA9 expression, and have identified the ZEB1-CA9 axis as a mediator of resistance to chemotherapy. ZEB1-CA9 mediates chemoresistance mainly via maintaining the $\mathrm{pHi}$ in response to chemotherapy, providing new insights into the complex mechanisms of drug resistance. Our findings strongly suggest that the ZEB1-CA9 axis can be a prognostic biomarker for tongue cancer patients and provides a rationale for the development of anticancer intervention strategies targeting the ZEB1-CA9 axis in clinics.

\section{Materials and methods}

\section{Cell culture and tissue specimens}

The human tongue squamous cell carcinoma cell line Tca8113 derived from moderately differentiated human tongue squamous cell carcinoma and the stable PYM-resistant cell line Tca8113/PYM, and squamous cell carcinoma cell line SCC-25 from American Type Culture Collection, were cultured in RPMI-1640 (Gibco) containing $10 \%$ fetal bovine serum (Gibco) at $37^{\circ} \mathrm{C}$ in a humidified atmosphere containing $5 \% \mathrm{CO}_{2}$. To maintain the resistance phenotype, $0.5 \mathrm{mg} / \mathrm{L}$ PYM was added to the culture media of Tca8113/PYM cells. The HEK293T human embryonic kidney cell line was cultured in DMEM (Gibco) with $10 \%$ fetal bovine serum. Eighty-four tongue cancer tissue specimens were obtained from patients at the Affiliated Tumor Hospital of Guangzhou Medical University between March 2000-December 2006. Overall survival was computed from the day of surgery to the day of death or of last follow-up. The study was approved by the ethics committee of the Affiliated Tumor Hospital of Guangzhou Medical University.

\section{ChIP assay}

The ChIP assay was performed using the EZ-CHIP ${ }^{\mathrm{m}}$ chromatin immunoprecipitation kit (Merck Millipore). Briefly: Chromatin proteins were cross-linked to DNA by addition of formaldehyde to the culture medium to a final concentration of $1 \%$. After a 10 min incubation at room temperature, the cells were washed and scraped off in ice-cold phosphate-buffered saline (PBS) containing Protease Inhibitor Cocktail II. Cells were pelleted and then resuspended in lysis buffer containing Protease Inhibitor Cocktail II. The resulting lysate was subjected to sonication to reduce the size of DNA to approximately 200-1000 base pairs in length. The sample was centrifuged to remove cell debris and diluted ten-fold in ChIP dilution buffer containing Protease Inhibitor Cocktail II. A $5 \mu$ sample of the supernatant was retained as "Input" and stored at $4^{\circ} \mathrm{C}$. Then $5 \mu \mathrm{g}$ of anti-RNA Polymerase antibody (positive control, included with the kit), or anti-ZEB1 antibody (cell signal technology) were added to the chromatin solution and incubated overnight at $4^{\circ} \mathrm{C}$ with rotation. After antibody incubation, protein $\mathrm{G}$ agarose was added and the sample incubated at $4^{\circ} \mathrm{C}$ with rotation for an additional 2 
h. The protein/DNA complexes were washed with Wash Buffers four times and eluted with ChIP Elution Buffer. Cross-links were then reversed to free DNA by the addition of $5 \mathrm{M} \mathrm{NaCl}$ and incubation at $65^{\circ} \mathrm{C}$ for $4 \mathrm{~h}$. The DNA was purified according to the manufacturer's instructions. $50 \mu \mathrm{l}$ of DNA was obtained for each treatment. $0.2 \mu \mathrm{l}$ of DNA from each group was used as a template for PCR. Primers for the $C A 9$ promoter containing putative ZEB1 binding sites were as follows, sense: 5'TGTTGGCCAGGCTGGTCT-3', antisense: 5'-CCACTT GAGTGCCTCAGCCA-3' (for site A); sense: 5' -GTAGTA TACCAGTCAGGTGTC-3', antisense: 5'-AGAGATGGG ATCTCACTGTGTT-3' (for site B); sense: 5'-CAGTCTC AGCTCACTGCAGC-3', antisense: 5'-GTGGGTGGTTG CTTGAGTCCAG-3' (for site C); sense: 5'-CTGACACAT ACACTTGCTTTTC-3', antisense: 5' -TGGCTGAGAGG GAAAGCAGCTC-3' (for site D); sense: 5'-CCTGCA TAGTGCCAGGTGG-3', antisense: 5'-GAGATGGAGC CAAAGTCTCACAG-3' (for site E). Primers for the human GAPDH gene: sense, 5'-TACTAGCGGTTT TACGGGCG-3', antisense, 5' -TCGAACAGGAGGAGCA GAGAGCGA-3'. The PCR conditions were as follows: 1 cycle of $95^{\circ} \mathrm{C}$ for $5 \mathrm{~min} ; 32$ cycles of $95^{\circ} \mathrm{C}$ for $20 \mathrm{~s}, 59^{\circ} \mathrm{C}$ for $30 \mathrm{~s}$, and $72^{\circ} \mathrm{C} 30 \mathrm{~s}$; and 1 cycle of $72^{\circ} \mathrm{C}$ for $10 \mathrm{~min}$. PCR samples were resolved by electrophoresis in a $2 \%$ agarose gel and stained with ethidium bromide.

\section{Cells transfection}

Cells were trypsinized, counted and seeded into six-well plates the day before transfection to ensure $70 \%$ cell confluency on the day of transfection. The transfection of the pLEX-ZEB1 vector, the pLEX-CA9 vector and related controls was carried out using Lipofectamine 2000 (Invitrogen) in accordance with the manufacturer's instructions. siRNAs targeting ZEB1 or CA9 and siRNA controls were purchased from Santa Cruz Biotechnology. Transfection of siRNA (50 $\mathrm{nM}$ final concentration) was performed as above. Experiments were performed $48 \mathrm{~h}$ post-transfection.

\section{Real-time PCR}

Total RNA was extracted using Trizol, and cDNAs subsequently synthesized from mRNA templates using the Super-Script first-strand synthesis system (Thermo Scientific). Real-time PCR was carried out according to standard protocols using an ABI 7500 with SYBR Green detection (Applied Biosystems). GAPDH was used as an internal control and the qRT-PCR was repeated three times. The primers for GAPDH were: forward primer 5'-ATTCCATGGCACCGTCAAGGCTGA-3', reverse primer 5'-TTCTCCATGGTGGTGAAGACGCCA-3'; primers for CA9 were: forward primer 5'- GTCCAGCTGAATTC CTGCCT-3', reverse primer $5^{\prime}$-CCTTCTGTGCTGCCT TCTCA-3'.

\section{Western blotting}

Total protein was extracted from cells using RIPA buffer (Pierce) in the presence of protease inhibitors (Protease Inhibitor Cocktail, Pierce). The protein concentration of lysates was measured using a BCA Protein Assay Kit (Pierce). Equivalent amounts of protein were mixed with $5 \times$ Lane Marker Reducing Sample Buffer (Pierce), and resolved by electrophoresis in a 10\% SDS-polyacrylamide gel and then transferred onto Immobilon-P Transfer Membrane (Millipore). The membranes were blocked with 5\% non-fat milk in Tris-buffered saline and then incubated with primary antibodies followed by secondary antibody. The signal was detected on the Odyssey instrument (LI-COR Bioscience). ZEB1, CA9, Caspase-3, and $\beta$-Actin antibodies were from Cell Signaling Technology, and the fluorescently labeled secondary antibodies were from LI-COR Bioscience.

\section{Promoter activity analysis}

To determine whether ZEB1 regulates the promoter activity of CA9, a three kilobase region upstream of the first exon of CA9 was cloned into the pGL4-reporter vector upstream of the luciferase gene. Cells were seeded in 96-well plates and co-transfected with the pGL4-reporter vector and the pRL-TK Renilla luciferase vector with or without the pLEX-ZEB1 vector using Lipofectamine 2000 (Invitrogen). After $48 \mathrm{~h}$, luciferase activity was determined using a Dual-Luciferase Reporter Assay System (Promega) on the BioTek Synergy 2. Renilla luciferase activity was used as an internal control and the firefly luciferase activity was calculated as the mean $+/$ - standard deviation after normalization relative to the Renilla luciferase activity.

\section{Cell proliferation assays (MTS)}

The CellTiter 96 AQueous One Solution Cell Proliferation Assay kit (Promega, Madison, WI, USA) was used to determine the sensitivity of cells to PYM or cDDP. Briefly, cells were seeded in 96-well plates at a density of $4 \times 10^{3}$ cells/well $(0.2 \mathrm{ml} /$ well $)$ for $24 \mathrm{~h}$ before use. The culture medium was replaced with fresh medium containing PYM or cDDP at different concentrations and cells were then incubated for a further $72 \mathrm{~h}$. Then, MTS $(0.02 \mathrm{ml} /$ well) was added. After a further $2 \mathrm{~h}$ incubation, the absorbance at $490 \mathrm{~nm}$ was recorded for each well on the BioTek Synergy 2. The absorbance represented the cell number and was used for the plotting of dose-cell number curves.

\section{Intracellular $\mathrm{pH}(\mathrm{pHi})$ measurement}

The pHi of cells was measured using the fluorescent $\mathrm{pH}$ indicator 2,7-bicarboxyethyl-5,6-carboxyfluorescein-acetoxymethylester (BCECF-AM) (Molecular Probes, Eugene, OR, USA) following the manufacturer's protocol. BCECF is a neutral lipophilic form of bis-carboxyfluorescein which 
diffuses freely through the plasma membrane. Within the cell it is hydrolyzed by esterases, releasing BCECF which is retained within the cytoplasm. The fluorescence intensity of BCECF is dependent upon pH. After an incubation of $10 \mathrm{~min}$, the fluorescence intensity was measured using confocal microscopy using an excitation wavelength of $535 \mathrm{~nm}$.

\section{Hoechst staining}

Following transfection cells were reseeded in fresh medium in 24-well plates. After a $24 \mathrm{~h}$ incubation, cells were treated with or without PYM $(80 \mathrm{mg} / \mathrm{L})$ or $\mathrm{cDDP}$ $(5 \mathrm{mg} / \mathrm{L})$ for an additional $48 \mathrm{~h}$. The cells were then stained with hoechst33528, and apoptotic cells possessing significantly smaller, condensed and fragmented nuclei, were observed using a fluorescence microscope. The apoptotic cell number was determined for at least three fields-of-view for each treatment and the apoptotic rate then calculated.

\section{Caspase- 3 activity assay}

Caspase-3 activity was determined using a caspase-3 activity kit (Beyotime), in which cleavage of a colorless substrate specific for caspase-3 [Ac-DEVD-p-nitroaniline (pNA)], releases the chromophore pNA. Assays were carried out according to the manufacturer's instructions. To evaluate the activity of caspase-3, lysates were prepared from cells after their respective treatments. Assays were performed in 96-well microtiter plates. Briefly; for each sample $10 \mu \mathrm{l}$ of cell lysate was combined with $80 \mu \mathrm{l}$ of reaction buffer and $10 \mu \mathrm{l}$ of caspase-3 substrate (Ac-DEVD-pNA, 2mm). Lysates were then incubated at $37^{\circ} \mathrm{C}$ for $4 \mathrm{~h}$ and samples subsequently measured on the BioTek Synergy 2 system using an absorbance of $405 \mathrm{~nm}$.

\section{Immunohistochemistry}

A tissue array containing 84 human tongue cancer specimens was cut into $4-\mu \mathrm{m}$ sections. The sections were dried at $62^{\circ} \mathrm{C}$ for $2 \mathrm{~h}$ and then deparaffinized in xylene and rehydrated using a series of graded alcohol washes. The tissue slides were then treated with $3 \%$ hydrogen peroxide in methanol for $15 \mathrm{~min}$ to quench endogenous peroxidase activity, and antigen retrieval then performed by incubation in $0.01 \mathrm{M}$ sodium cirate buffer ( $\mathrm{pH}$ 6.0) and heating using a microwave oven. After a $1 \mathrm{~h}$ preincubation in $10 \%$ goat serum, the specimens were incubated with primary antibody overnight at $4^{\circ} \mathrm{C}$. The tissue slides were treated with a non-biotin horseradish peroxidase detection system according to the manufacturer's instruction (DAKO). Two different pathologists evaluated the immunohistological samples.

\section{Statistical analysis}

All statistical analyses were performed with SPSS statistical software (version 21.0; IBM). Survival curves were constructed using the Kaplan-Meier method and analyzed by the log-rank test. Significant prognostic factors identified by univariate analysis were entered into multivariate analysis using the Cox proportional hazards model. The Student's t-test was used for comparisons and the Pearson correlation test (two-tailed) was used to investigate the correlation between ZEB1 and CA9 protein levels. Statistical significance was defined as $\mathrm{P}<0.05$.

\section{Competing interests}

The authors declare that they have no competing interests.

\section{Authors' contributions}

GZ, CW and ZH designed the experiments. GZ, CP, XJ, ZZ, YD, NL, JY, XL and ML performed the experiments. YG and HT analyzed the data. GZ, NL and $\mathrm{ZH}$ wrote the manuscript. All the authors approved the final draft of this manuscript.

\section{Acknowledgments}

This study was supported by grants from National Natural Science Foundation (No. 30873088) of China, National Natural Science Foundation (81272450) of China, Natural Science Foundation of Gongdong Province (S2012010008995) and Doctoral Fund of Ministry of Education of China (20124423110003).

\section{Author details}

${ }^{1}$ Cancer Hospital and Cancer Research Institute of Guangzhou Medical University, Hengzhigang Road 78\#, Guangzhou 510095, Guangdong, China. ${ }^{2}$ Department of Breast Oncology, Sun Yat-Sen University Cancer Center, State Key Laboratory of Oncology in South China, Collaborative Innovation Center for Cancer Medicine, East Dongfeng Road 651\#, Guangzhou 510060, Guangdong, China.

Received: 30 July 2014 Accepted: 2 April 2015

Published online: 15 April 2015

\section{References}

1. Jemal A, Bray F, Center MM, Ferlay J, Ward E, Forman D. Global cancer statistics. CA Cancer J Clin. 2011;61:69-90.

2. Toner M, O'Regan EM. Head and neck squamous cell carcinoma in the young: a spectrum or a distinct group? Part 2. Head Neck Pathol. 2009;3:249-51.

3. Gibson MK, Li Y, Murphy B, Hussain MH, DeConti RC, Ensley J, et al. Randomized phase III evaluation of cisplatin plus fluorouracil versus cisplatin plus paclitaxel in advanced head and neck cancer (E1395): an intergroup trial of the Eastern Cooperative Oncology Group. J Clin Oncol. 2005;23:3562-7.

4. Sève $P$, Isaac $S$, Trédan $O$, Souquet $P J$, Pachéco $Y$, Pérol $M$, et al. Expression of class III \{beta\}-tubulin is predictive of patient outcome in patients with non-small cell lung cancer receiving vinorelbine-based chemotherapy. Clin Cancer Res. 2005;11:5481-6.

5. Yamauchi $K$, Yang M, Hayashi K, Jiang P, Yamamoto N, Tsuchiya $H$, et al. Induction of cancer metastasis by cyclophosphamide pretreatment of host mice: an opposite effect of chemotherapy. Cancer Res. 2008;68:516-20.

6. Broxterman HJ, Gotink KJ, Verheul HM. Understanding the causes of multidrug resistance in cancer: a comparison of doxorubicin and sunitinib. Drug Resist Updat. 2009;12:114-26.

7. Zheng G, Zhou M, Ou X, Peng B, Yu Y, Kong F, et al. Identification of carbonic anhydrase 9 as a contributor to pingyangmycin-induced drug resistance in human tongue cancer cells. FEBS J. 2010;277:4506-18.

8. Koukourakis MI, Giatromanolaki A, Sivridis E, Simopoulos K, Pastorek J, Wykoff CC, et al. Hypoxia-regulated carbonic anhydrase-9 (CA9) relates to poor vascularization and resistance of squamous cell head and neck cancer to chemoradiotherapy. Clin Cancer Res. 2001;7:3399-403. 
9. Pastorekova S, Zatovicova M, Pastorek J. Cancer associated carbonic anhydrases and their inhibition. Curr Pharm Des. 2008;14:685-98.

10. Pouyssegur J, Dayan F, Mazure NM. Hypoxia signaling in cancer and approaches to enforce tumour regression. Nature. 2006;441:437-43.

11. Brahimi-Horn MC, Chiche J, Pouyssegur J. Hypoxia signalling controls metabolic demand. Curr Opin Cell Biol. 2007;19:223-9.

12. Roos A, Boron WF. Intracelluar pH. Physiol Rev. 1981;61:296-434.

13. Pouyssegur J, Sardet C, Franchi A, L'Allemain G, Paris S. A specific mutation abolishing $\mathrm{Na}+/ \mathrm{H}+$ antiport activity in hamster fibroblasts precludes growth at neutral and acidic pH. Proc Natl Acad Sci USA. 1984;81:4833-7.

14. Chambard JC, Pouyssegur J. Intracellular pH controls growth factor-induced ribosomal protein S6 phosphorylation and protein synthesis in the G0-G1 transition of fibroblasts. Exp Cell Res. 1986;164:282-94.

15. Wykoff CC, Beasley NJ, Watson PH, Turner KJ, Pastorek J, Sibtain A, et al. Hypoxia-inducible expression of tumor-associated carbonic anhydrases. Cancer Res. 2000;60:7075-83.

16. Robertson N, Potter C, Harris AL. Role of carbonic anhydrase IX in human tumor cell growth, survival, and invasion. Cancer Res. 2004;64:6160-5.

17. Park HJ, Makepeace CM, Lyons JC, Song CW. Effect of intracellular acidity and ionomycin on apoptosis in HL-60 cells. Eur J Cancer. 1996;32A:540-6.

18. Rich IN, Worthington-White D, Garden OA, Musk P. Apoptosis of leukemic cells accompanies reduction in intracellular $\mathrm{pH}$ after targeted inhibition of the $\mathrm{Na}(+) / \mathrm{H}(+)$ exchanger. Blood. 2000;95:1427-34.

19. Merlo LM, Pepper JW, Reid BJ, Maley CC. Cancer as an evolutionary and ecological process. Nat Rev Cancer. 2006;6:924-35.

20. Schmalhofer O, Brabletz S, Brabletz T. E-cadherin, beta-catenin, and ZEB1 in malignant progression of cancer. Cancer Metastasis Rev. 2009;28:151-66.

21. Gregory PA, Bert AG, Paterson EL, Barry SC, Tsykin A, Farshid G, et al. The miR-200 family and miR-205 regulate epithelial to mesenchymal transition by targeting ZEB1 and SIP1. Nat Cell Biol. 2008;10:593-601.

22. Wellner U, Schubert J, Burk UC, Schmalhofer O, Zhu F, Sonntag A, et al. The EMT-activator ZEB1 promotes tumorigenicity by repressing stemness-inhibiting microRNAs. Nat Cell Biol. 2009;11:1487-95.

23. Ren J, Chen Y, Song H, Chen L, Wang R. Inhibition of ZEB1 reverses EMT and chemoresistance in docetaxel-resistant human lung adenocarcinoma cell line. J Cell Biochem. 2013;114:1395-403.

24. Siebzehnrubl FA, Silver DJ, Tugertimur B, Deleyrolle LP, Siebzehnrubl D, Sarkisian MR, et al. The ZEB1 pathway links glioblastoma initiation, invasion and chemoresistance. EMBO Mol Med. 2013;5:1196-212.

25. Zhang P, Wei Y, Wang L, Debeb BG, Yuan Y, Zhang J, et al. ATM-mediated stabilization of ZEB1 promotes DNA damage response and radioresistance through CHK1. Nat Cell Biol. 2014;16:864-75.

26. Zhang $P$, Wang L, Rodriguez-Aguayo C, Yuan Y, Debeb BG, Chen D, et al. miR-205 acts as a tumour radiosensitizer by targeting ZEB1 and Ubc13. Nat Commun. 2014;5:5671.

27. Parks SK, Chiche J, Pouyssegur J. pH control mechanisms of tumor survival and growth. J Cell Physiol. 2011;226:299-308

28. Neri $\mathrm{D}$, Supuran $\mathrm{CT}$. Interfering with $\mathrm{pH}$ regulation in tumours as a therapeutic strategy. Nat Rev Drug Discov. 2011;10:767-77.

29. McDonald PC, Winum JY, Supuran CT, Dedhar S. Recent developments in targeting carbonic anhydrase IX for cancer therapeutics. Oncotarget. 2012;3:84-97.

30. Vincent AM, TenBroeke M, Maiese K. Neuronal intracellular pH directly mediates nitric oxide-induced programmed cell death. J Neurobiol. 1999;40:171-84.

31. Matsuyama S, Llopis J, Deveraux QL, Tsien RY, Reed JC. Changes in intramitochondrial and cytosolic pH: early events that modulate caspase activation during apoptosis. Nat Cell Biol. 2000;2:318-25.

32. Chiche J, Ilc K, Laferrie're J, Trottier E, Dayan F, Mazure NM, et al. Hypoxia-inducible carbonic anhydrase IX and XII promote tumor cell growth by counteracting acidosis through the regulation of the intracellular $\mathrm{pH}$. Cancer Res. 2009;69:358-68.

33. Swietach P, Patiar S, Supuran CT, Harris AL, Vaughan-Jones RD. The role of carbonic anhydrase 9 in regulating extracellular and intracellular ph in three-dimensional tumor cell growths. J Biol Chem. 2009;284:20299-310.

34. Swietach P, Hulikova A, Vaughan-Jones RD, Harris AL. New insights into the physiological role of carbonic anhydrase IX in tumour pH regulation. Oncogene. 2010;29:6509-21.

\section{Submit your next manuscript to BioMed Central and take full advantage of:}

- Convenient online submission

- Thorough peer review

- No space constraints or color figure charges

- Immediate publication on acceptance

- Inclusion in PubMed, CAS, Scopus and Google Scholar

- Research which is freely available for redistribution

Submit your manuscript at www.biomedcentral.com/submit 